\title{
The bifurcation of homoclinic orbits of maps of the interval
}

\author{
LOUIS BLOCK AND DAVID HART
}

Department of Mathematics, University of Florida, Gainesville, Florida 32611, USA

(Received 14 June 1982)

Abstract. Relationships involving homoclinic orbits of various periods and the Sarkovskii stratification are given and corresponding bifurcation properties are derived. It is shown that if a continuous map has one homoclinic periodic orbit, it has infinitely many. In any family of $C^{1}$ maps going from zero to positive entropy, infinitely many homoclinic bifurcations occur, involving periods which are successively smaller powers of two.

\section{Introduction}

The presence of homoclinic periodic points has been shown to be equivalent to chaotic dynamics, i.e. horseshoes or positive topological entropy for maps of the interval ([1], [4]). In this paper we investigate the structure of the set of continuous mappings of the interval which have homoclinic periodic points, and derive bifurcation theorems for continuous families in $C^{1}(I, I)$ which include such maps. Here $I$ denotes a compact interval.

A periodic point $p$ of (least) period $k$ under $f$ is said to be homoclinic if

$$
p \in f^{k}\left(W^{u}\left(p, f^{k}\right) \backslash\{p\}\right) \text {. }
$$

Recall that the unstable manifold of $p$ under $f^{k}, W^{u}\left(p, f^{k}\right)$, is defined to be the set of $x \in I$ such that for every neighbourhood $U$ of $p$,

$$
x \in f^{k n}(U)
$$

for some positive integer $n$. We define $F(k)$ to be the set of continuous mappings of $I$ to itself with a periodic point of period $k$ and $H(k)$ to be the set of continuous mappings of $I$ to itself with a homoclinic periodic point of period $k$. Thus [6] shows that $F(m) \subseteq F(n)$ if $n \triangleleft m$ in the Sarkovskii order

$$
1 \triangleleft 2 \triangleleft \cdots \triangleleft 5 \cdot 4 \triangleleft 3 \cdot 4 \triangleleft \cdots \triangleleft 5 \cdot 2 \triangleleft 3 \cdot 2 \triangleleft \cdots \triangleleft 5 \triangleleft 3 \text {, }
$$

and [1] shows that $f \in F(n)$ for some $n$ not a power of two if and only if $f$ has a homoclinic periodic point of some period. In particular it follows from [1] that $F(3) \subseteq H(1) \cup H(2)$.

We begin with a generalization of this result. Let $H^{\prime}(n)$ denote the set of maps $f \in C^{0}(I, I)$ such that $f^{n}$ has a homoclinic fixed point. Note that for any positive integer $k$,

$$
H^{\prime}\left(2^{k}\right)=H(1) \cup H(2) \cup H(4) \cup \cdots \cup H\left(2^{k}\right)
$$

(see proposition 1 for details). 
THEOREM 1. For every odd integer $m>1, F(m) \subseteq H^{\prime}(2)$. Hence, for any non-negative integer $r$ and odd integer $m>1$,

$$
F\left(m \cdot 2^{r}\right) \subseteq H^{\prime}\left(2^{r+1}\right) \text {. }
$$

Our next result shows that if $x$ has one homoclinic periodic point then $f$ has infinitely many (of infinitely many periods).

THEOREM 2. $H(1) \subseteq H(2) \cup H(4)$. Hence, for any non-negative integer $r$,

$$
H\left(2^{r}\right) \subseteq H\left(2^{r+1}\right) \cup H\left(2^{r+2}\right)=H^{\prime}\left(2^{r+2}\right) .
$$

We remark that smooth examples in $H(1) \backslash H(2)$ are easily constructed, so that

$$
H\left(2^{r}\right) \subseteq H\left(2^{r+1}\right)
$$

can only be expected under additional hypothesis (for example for one-humped maps with negative Schwarzian derivatives).

THEOREM 3. $H(1) \subseteq F(6)$. Hence,

for any non-negative integer $r$.

$$
H\left(2^{r}\right) \subseteq F\left(3 \cdot 2^{r+1}\right)
$$

Theorem 3 is also sharp in the sense that if $f \in H(1)$, the existence of periodic points of $f$ of odd period is not necessary. Using theorems 1,2 and 3 , and the result of [2] we obtain:

THEOREM 4. $H\left(2^{k}\right) \subseteq \operatorname{int}\left(H\left(2^{k+1}\right) \cup H\left(2^{k+2}\right)\right)$ for any non-negative integer $k$.

In theorem 4 , int denotes the interior of the set in $C^{0}(I, I)$. Note that in all of the previous results, no differentiability is assumed. Our final two results, however, are valid only in the differentiable case.

THEOREM 5. If $f$ is in the closure in $C^{1}(I, I)$ of the set of $C^{1}$ maps with a homoclinic fixed point then $f^{2}$ has a homoclinic fixed point. Hence, for any non-negative integer $r$,

$$
\overline{H^{\prime}\left(2^{\prime}\right) \cap C^{1}(I, I)} \subseteq H^{\prime}\left(2^{r+1}\right) \cap C^{1}(I, I) .
$$

We remark that one can construct a map $f$ satisfying the hypothesis of the first assertion of theorem 5 so that $f$ does not have a homoclinic fixed point.

Our final result implies that for any one parameter family of $C^{1}$ maps going from zero to positive entropy an infinite sequence of bifurcations occur, where new homoclinic periodic orbits (of period successively smaller powers of two) are created.

THEOREM 6. If $f_{t}$ is a continuous arc in $C^{1}(I, I), 0 \leq t \leq 1$, such that $f_{0}$ has no homoclinic periodic point and $f_{1} \in H\left(2^{m}\right)$ for some non-negative integer $m$, then for every integer $n>m$, there exists $t(n) \in(0,1)$ such that

$$
f_{t(n)} \in H^{\prime}\left(2^{n+1}\right) \backslash H^{\prime}\left(2^{n-1}\right)=\left(H\left(2^{n}\right) \cup H\left(2^{n+1}\right)\right) \backslash\left(H(1) \cup H(2) \cup \cdots \cup H\left(2^{n-1}\right)\right) .
$$

\section{Proofs}

We now proceed with our proofs. The proof of theorem 1 is essentially that of theorem 5 of [1] but is included for completeness (see also lemma 2.5 of [5]). We begin with a lemma which will be used several times. 
LEMMA 1. If $f \in C^{0}(I, I)$ and $f$ has a homoclinic fixed point $p$ then (at least) one of the following holds:

(1) There are points $y$ and $z$ in $W^{u}(p, f)$ with $p<y<z$, such that $f(y)=z$ and $f(z)=p$.

(2) There are points $y$ and $z$ in $W^{u}(p, f)$ with $z<y<p$, such that $f(y)=z$ and $f(z)=p$.

(3) There are points $z_{0}, z_{1}, z_{2}, \ldots$, in $W^{u}(p, f)$ with

$$
z_{1}<z_{3}<z_{5}<\cdots<p<\cdots<z_{4}<z_{2}<z_{0}
$$

such that $f\left(z_{n}\right)=z_{n-1}$ for every positive integer $n, f\left(z_{0}\right)=p$, and $p=\lim _{n \rightarrow \infty} z_{n}$.

(4) There are points $z_{0}, z_{1}, z_{2}, \ldots$, in $W^{u}(p, f)$ with

$$
z_{0}<z_{2}<z_{4}<\cdots<p<\cdots<z_{5}<z_{3}<z_{1}
$$

such that $f\left(z_{n}\right)=z_{n-1}$ for every positive integer $n, f\left(z_{0}\right)=p$, and $p=\lim _{n \rightarrow \infty} z_{n}$.

Proof. Suppose (1) and (2) do not hold. Since $p$ is a homoclinic fixed point of $f$, for some

$$
z \in W^{u}(p, f)
$$

with $z \neq p, f(z)=p$. Suppose that $z>p$ (the other case is similar).

Since (1) does not hold, $z \notin f(p, z)$. Hence, since

$$
z \in W^{u}(p, f)
$$

it follows that $f(y)=z$ for some $y<p$. We take the maximal $y$ with $y<p$ and $f(y)=z$. Then

$$
z \in W^{u}(p, f)
$$

implies that

$$
y \in W^{u}(p, f)
$$

Since (2) does not hold,

$$
y \notin f(y, p) \text {. }
$$

(This is true because if $y=f(x)$ for some $x \in(y, p)$, then for some $\tilde{z} \in(y, x)$,

$$
f(\tilde{z})=p
$$

and for some $\tilde{y} \in(x, p)$,

$$
f(\tilde{y})=\tilde{z}
$$

so (2) holds.) Hence, since

$$
y \in W^{u}(p, f)
$$

it follows that $f(x)=y$ for some $x>p$. We may take the minimal $x$ with $x>p$ and $f(x)=y$. Then $p<x<z$ (because if $x>z$ then $y \notin f(y, z)$, which contradicts

$$
\left.y \in W^{u}(p, f)\right) \text {. }
$$

Let $z_{0}=z, z_{1}=y$, and $z_{2}=x$. By induction we obtain $z_{3}, z_{4}, \ldots$, with

$$
z_{1}<z_{3}<z_{5}<\cdots<p<\cdots<z_{4}<z_{2}<z_{0}
$$

and $f\left(z_{n}\right)=z_{n-1}$ for every positive integer $n$. The sequences $\left(z_{2 n+1}\right)$ and $\left(z_{2 n}\right)$ are increasing and decreasing respectively, so $\left(z_{2 n+1}\right)$ converges to $a$ and $\left(z_{2 n}\right)$ converges 
to $b$ with $a \leq p \leq b$. If either $a \neq p$ or $b \neq p$ then $a<p<b$, and (using the maximality of $z_{2 n+1}$ and the minimality of $z_{2 n}$ )

$$
f([a, b]) \subseteq[a, b] .
$$

This contradicts the fact that the $z_{n}$ are in $W^{u}(p, f)$. Hence $a=b=p$ and $p=$ $\lim _{n \rightarrow \infty} z_{n}$.

Proposition 1. Let $n=m t$ (where $n, m$, and $t$ are positive integers) and let $p$ be a periodic point of $f \in C^{0}(I, I)$ of period $t$. Then $p$ is a homoclinic periodic point of $f$ if and only if $p$ is a homoclinic fixed point of $f^{n}$. In particular, for any positive integer $k$,

$$
H^{\prime}\left(2^{k}\right)=H(1) \cup H(2) \cup H(4) \cup \cdots \cup H\left(2^{k}\right) .
$$

Proof. It suffices to prove the first statement. Recall that by definition, $p$ is a homoclinic periodic point if and only if $p$ is a homoclinic fixed point $f^{t}$. Thus, we must show that

$$
p \in f^{t}\left(W^{u}\left(p, f^{t}\right) \backslash\{p\}\right)
$$

if and only if $p \in f^{n}\left(W^{u}\left(p, f^{n}\right) \backslash\{p\}\right)$.

First suppose that

$$
p \in f^{n}\left(W^{u}\left(p, f^{n}\right) \backslash\{p\}\right) .
$$

For some $z \in W^{u}\left(p, f^{n}\right)$ with $z \neq p, f^{n}(z)=p$. Then $z \in W^{u}\left(p, f^{t}\right)$ (as $W^{u}\left(p, f^{n}\right) \subseteq$ $\left.W^{u}\left(p, f^{t}\right)\right)$ and $f^{m t}(z)=p$. Since $W^{u}\left(p, f^{t}\right)$ is invariant under $f^{t}$, each of the points $z, f^{t}(z), f^{2 t}(z), \ldots, f^{(m-1) t}(z)$ is in $W^{u}\left(p, f^{t}\right)$. Hence,

$$
p \in f^{t}\left(W^{u}\left(p, f^{t}\right) \backslash\{p\}\right) .
$$

Now suppose that

$$
z \in f^{t}\left(W^{u}\left(p, f^{t}\right) \backslash\{p\} .\right.
$$

Let $V$ be any neighbourhood of $p$. It follows from lemma 1 (applied to $f^{t}$ ) that there is a point $z \in W^{u}\left(p, f^{t}\right)$ with $z \neq p$ and $f^{t}(z)=p$, such that $z \in f^{i t}(V)$ for all sufficiently large integers $i$. In particular, $z \in f^{\text {in }}(V)$ for some positive integer $j$. Thus,

$$
z \in W^{u}\left(p, f^{n}\right) \text {. }
$$

Since $z \neq p$ and $f^{n}(z)=p$, it follows that

$$
p \in f^{n}\left(W^{u}\left(p, f^{n}\right) \backslash\{p\}\right) .
$$

THEOREM 1. For every odd integer, $m>1, F(m) \subseteq H^{\prime}(2)$. Hence, for any non-negative integer $r$ and odd integer $m>1, F\left(m \cdot 2^{r}\right) \subseteq H^{\prime}\left(2^{r+1}\right)$.

Proof. It suffices to prove the first statement, so let $f \in F(m)$ where $m$ is odd and $m>1$. We may assume without loss of generality that $f \notin F(k)$ for any odd $k$ with $1<k<m$. Hence, $f$ has a periodic orbit $\left\{p_{1}, p_{2}, \ldots, p_{m}\right\}$ of period $m$ which can be numbered such that $f\left(p_{i}\right)=p_{i+1}$ for $1 \leq i<m, f\left(p_{m}\right)=p_{1}$, and the $p_{i}$ lie in the order

$$
p_{m}<p_{m-2}<\cdots<p_{1}<p_{2}<p_{4}<\cdots<p_{m-1},
$$

or in the mirror image [7]; assume they lie in the order given. Since

$$
f^{2}\left(\left[p_{1}, p_{2}\right]\right) \supseteq\left[p_{1}, p_{2}\right],
$$


$f^{2}$ has a fixed point in the interval $\left(p_{1}, p_{2}\right)$. Let $q$ be the minimal such point. Then

$$
p_{1} \in W^{u}\left(q, f^{2}\right)
$$

since $f^{2}(x)<x$ for all $x \in\left[p_{1}, q\right]$. Since $W^{u}\left(q, f^{2}\right)$ is $f^{2}$-invariant, and $m$ is odd,

$$
\left[p_{m}, p_{m-1}\right] \subseteq W^{u}\left(q, f^{2}\right)
$$

But

$$
f^{2}\left(\left[p_{m}, p_{m-2}\right]\right) \supseteq\left[p_{m}, p_{2}\right],
$$

so $q=f^{2}(x)$ for some $x \in\left[p_{m}, p_{m-2}\right]$. Thus $x \neq q$ and $x \in W^{u}\left(q, f^{2}\right)$, and $q$ is a homoclinic fixed point of $f^{2}$.

THEOREM 2. $H(1) \subseteq H(2) \cup H(4)$. Hence, for any non-negative integer $r$,

$$
H\left(2^{r}\right) \subseteq H\left(2^{r+1}\right) \cup H\left(2^{r+2}\right)=H^{\prime}\left(2^{r+2}\right) \text {. }
$$

Proof. Let $f \in H(1)$. One of the cases stated in lemma 1 must hold; since cases (1) and (2) are analogous, and (3) and (4) are analogous, it suffices to consider (1) and (3).

First suppose that (1) holds. We may assume that $y$ is the maximal point in $(p, z)$ with $f(y)=z$. For some $x \in(p, y), f(x)=y$, and we also assume that $x$ is maximal. Since

$$
f^{2}(x, y) \supseteq(p, z),
$$

$f^{4}$ has a fixed point $q$ in $(x, y)$, which we require to be minimal.

Suppose $f^{2}(q)=q$. Then

$$
f^{2}(x, q) \supseteq(q, z)
$$

which implies that for some $r \in(x, q), f^{2}(r)=y$. Hence

so

$$
f^{2}(x, r) \supseteq(y, z)
$$

$$
f^{4}(x, r) \supseteq(p, z) \supseteq(x, r) .
$$

Thus, $f^{4}$ has a fixed point in $(x, r)$, contradicting the minimality of $q$. Therefore $f^{2}(q) \neq q$ and $q$ is a periodic point of $f$ of period 4 .

Note finally that $f^{4}(x)<x, f^{4}(q)=q$, and $q$ minimal imply that $x \in W^{u}\left(q, f^{4}\right)$. Since $f^{4}(x)=p$,

$$
[p, x] \subseteq W^{u}\left(q, f^{4}\right)
$$

and $q \in f^{4}(p, x)$ is homoclinic. Thus, $f \in H(4)$, as desired.

Now, suppose that case (3) of lemma 1 holds. Then

$$
f^{2}\left(z_{2}, z_{0}\right) \supseteq\left(p, z_{0}\right)
$$

which implies that for some

$$
q \in\left(z_{2}, z_{0}\right), \quad f^{4}(q)=q .
$$

We again take the minimal such point. Then $f(q) \neq q$. To see this, suppose $f(q)=q$. Then

$$
f\left(z_{2}, q\right) \supseteq\left(z_{1}, q\right)
$$

and for some $r \in\left(z_{2}, q\right)$,

$$
f(r)=z_{2}
$$


Hence,

$$
f^{2}\left(z_{2}, r\right) \supseteq\left(z_{1}, z_{0}\right) \supseteq\left(z_{2}, r\right) .
$$

Thus $f^{4}$ has a fixed point in $\left(z_{2}, r\right)$, contradicting the minimality of $q$. This establishes our claim that $f(q) \neq q$, so $q$ is a periodic point of $f$ of period 2 or 4 .

Since, $f^{4}\left(z_{2}\right)=p<z_{2}$, and $q$ is minimal,

$$
[p, q] \subseteq W^{u}\left(q, f^{4}\right) .
$$

Since $q \in f^{4}\left(p, z_{4}\right), q$ is a homoclinic fixed point of $f^{4}$. It follows from proposition 1 that $q$ is a homoclinic periodic point of $f$ of period 2 or 4 . Thus, $f \in H(2) \cup H(4)$. This proves the first assertion of the theorem.

Finally, suppose that $f \in H\left(2^{r}\right)$ for some non-negative integer $r$, and let $n=2^{r}$. Then $f^{n}$ has a homoclinic fixed point, so $f^{n}$ has a homoclinic periodic point $q$ of period 2 or 4 . It follows that the period of $q$, as a periodic point of $f$, is $2 n=2^{r+1}$ or $4 n=2^{r+2}$. Hence,

$$
f \in H\left(2^{r+1}\right) \cup H\left(2^{r+2}\right) .
$$

This shows that

$$
H\left(2^{r}\right) \subseteq H\left(2^{r+1}\right) \cup H\left(2^{r+2}\right) .
$$

It follows by repeated application of this fact that

$$
H\left(2^{r+1}\right) \cup H\left(2^{r+2}\right)=H^{\prime}\left(2^{r+2}\right) .
$$

THEOREM 3. $H(1) \subseteq F(6)$. Hence,

$$
H\left(2^{r}\right) \subseteq F\left(3 \cdot 2^{r+1}\right)
$$

for any non-negative integer $r$.

Proof. It suffices to prove the first statement (in light of the theorem of Sarkovskii). Suppose $f$ has a homoclinic fixed point $p$. We may consider only two cases, (1) and (3), of lemma 1.

First, suppose that (1) holds. Then each of the intervals $f([p, y])$ and $f([y, z])$ contains $[p, z]$. Thus $f$ has a periodic point of period 3 , hence $f \in F(6)$.

Next suppose that (3) holds. Then each of the intervals $f^{2}\left(\left[p, z_{2}\right]\right)$ and $f^{2}\left(\left[z_{2}, z_{0}\right]\right)$ contains $\left[p, z_{0}\right]$. Thus $f^{2}$ has a periodic point of period 3 , and $f \in F(6)$.

THEOREM 4. $H\left(2^{k}\right) \subseteq \operatorname{int}\left(H\left(2^{k+1}\right) \cup H\left(2^{k+2}\right)\right)$ for any non-negative integer $k$.

Proof. We will use the fact that

$$
F\left(3 \cdot 2^{k+1}\right) \subseteq \operatorname{int}\left(F\left(5 \cdot 2^{k+1}\right)\right),
$$

see reference [2]. By theorem 3 ,

$$
H\left(2^{k}\right) \subseteq F\left(3 \cdot 2^{k+1}\right),
$$

and by theorem 1 ,

$$
F\left(5 \cdot 2^{k+1}\right) \subseteq H^{\prime}\left(2^{k+2}\right)
$$

By theorem 2,

$$
H^{\prime}\left(2^{k+2}\right)=H\left(2^{k+1}\right) \cup H\left(2^{k+2}\right)
$$


Thus,

$$
H\left(2^{k}\right) \subseteq \operatorname{int}\left(H\left(2^{k+1}\right) \cup H\left(2^{k+2}\right)\right) .
$$

THEOREM 5. If $f$ is in the closure in $C^{1}(I, I)$ of the set of maps with a homoclinic fixed point, then $f^{2}$ has a homoclinic fixed point. Hence, for any non-negative integer $r$,

$$
\overline{H^{\prime}\left(2^{r}\right) \cap C^{1}(I, I)} \subseteq H^{\prime}\left(2^{r+1}\right) \cap C^{1}(I, I) .
$$

Proof. It suffices to prove the first statement. Suppose $f$ is in the closure in $C^{1}(I, I)$ of the set of $C^{1}$ maps with a homoclinic fixed point. Then there is a sequence of maps $\left(f_{n}\right)$ such that each $f_{n}$ has a homoclinic fixed point $p_{n}$ and $\left(f_{n}\right)$ converges to $f$ in $C^{1}(I, I)$. Let $g_{n}=f_{n}^{2}$ and $g=f^{2}$. It follows from proposition 1 that $p_{n}$ is a homoclinic fixed point of $g_{n}$ for each $n$. Also, since $g_{n}=\left(f_{n}\right)^{2}$,

$$
\left(g_{n}\right)^{\prime}\left(p_{n}\right) \geq 0 \text {. }
$$

Hence, $\left(g_{n}\right)^{\prime}\left(p_{n}\right) \geq 1$, since otherwise

$$
W^{u}\left(p_{n}, g_{n}\right)=\left\{p_{n}\right\} \text {. }
$$

For each $n$, we may apply lemma 3 to $g_{n}$ and $p_{n}$ and conclude that (1), (2), (3), or (4) must hold. Since $g^{\prime}\left(p_{n}\right) \geq 1$, neither (3) nor (4) are possible. Since (1) and (2) are analogous, and one of these must hold for infinitely many $n$, we assume that (1) holds for all $n$.

Thus, for each $n$ there are points $y_{n}$ and $z_{n}$ with $p_{n}<y_{n}<z_{n}$, such that

$$
g_{n}\left(y_{n}\right)=z_{n} \text { and } g_{n}\left(z_{n}\right)=p_{n} \text {. }
$$

We may assume that $\left(p_{n}\right)$ converges to $p,\left(z_{n}\right)$ converges to $z$, and $\left(y_{n}\right)$ converges to $y$. Then $p \leq y \leq z, g(y)=z, g(z)=p, g(p)=p$, and $g^{\prime}(p) \geq 1$. Since $\left(g_{n}\right)^{\prime}\left(v_{n}\right)<0$ for some $v_{n}$ in the interval $\left(y_{n}, z_{n}\right)$ for each $n$, and $\left(g_{n}\right)$ converges to $g$ in $C^{1}(I, I)$, $p<y<z$.

Let $\tilde{p}$ be the largest fixed point of $g$ in $[p, y]$. Then $g(x)>x$ for all $x$ in $(\tilde{p}, y]$, so $y \in W^{u}(\tilde{p}, g)$. Since $g(y)=z$,

$$
[y, z] \subseteq W^{u}(\tilde{p}, g) .
$$

For some $\tilde{z} \in[y, z], g(\tilde{z})=\tilde{p}$, which is thus a homoclinic fixed point of $g=f^{2}$.

THEOREM 6. If $f_{t}$ is a continuous arc in $C^{1}(I, I), 0 \leq t \leq 1$, such that $f_{0}$ has no homoclinic periodic point and $f_{1} \in H\left(2^{m}\right)$ for some non-negative integer $m$, then for every integer $n>m$ there exists $t(n) \in(0,1)$ such that

$$
f_{t(n)} \in H^{\prime}\left(2^{n+1}\right) \backslash H^{\prime}\left(2^{n-1}\right)=\left(H\left(2^{n+1}\right) \cup H\left(2^{n}\right)\right) \backslash\left(H\left(2^{n-1}\right) \cup \cdots \cup H(2) \cup H(1)\right) .
$$

Proof. Let $s(n)=\inf \left\{s \in[0,1]: f_{s} \in H^{\prime}\left(2^{n+1}\right)\right\}$. Note that this set is non-empty, since $f_{1} \in H\left(2^{m}\right)$ and $n>m$. By theorem 4 and proposition 1 ,

If

$$
f_{s(n)} \notin H^{\prime}\left(2^{n-1}\right) \text {. }
$$

$$
f_{s(n)} \in H^{\prime}\left(2^{n+1}\right),
$$

the conclusion follows with $t(n)=s(n)$. Suppose

$$
f_{s(n)} \notin H^{\prime}\left(2^{n+1}\right) \text {. }
$$


Then

$$
f_{s(n)} \notin \overline{H^{\prime}\left(2^{n}\right)},
$$

by theorem 5 , and for $t(n)$ slightly larger than $s(n)$,

$$
f_{t(n)} \in H^{\prime}\left(2^{n+1}\right) \backslash H^{\prime}\left(2^{n}\right) \subseteq H^{\prime}\left(2^{n+1}\right) \backslash H^{\prime}\left(2^{n-1}\right) .
$$

\section{REFERENCES}

[1] L. Block. Homoclinic points of mappings of the interval. Proc. Amer. Math. Soc. 72 (1978), 576-580.

[2] L. Block. Stability of periodic orbits in the theorem of Sarkovskii. Proc. Amer. Math. Soc. 81 (1981), 333-336.

[3] L. Block \& D. Hart. The bifurcation of periodic orbits of one-dimensional maps. Ergod. Th. \& Dynam. Sys. 2 (1982), 125.

[4] M. Misiurewicz. Horseshoes for mappings of the interval. Bull. Acad. Polon. Sci. 27 (1979), 167-169.

[5] Z. Nitecki. Topological dynamics on the interval. Ergod. Theory and Dynamical Systems, Vol II (Proc. of special year, Maryland 1979-80), Progr. in Math. Birkhauser: Boston, 1981. (To appear.)

[6] A. Sarkovskii. Coexistence of cycles of a continuous map of the line into itself. Ukr. Mat. Z. 16 (1964), pp. 61-71. (In Russian.)

[7] P. Stefan. A theorem of Sarkovskii on the existence of periodic orbits of continuous endomorphisms of the real line. Comm. Math. Phys. 54 (1977), 237-248. 